\title{
Distributional framework for solving fractional differential equations
}

\author{
Teodor M. Atanackovic* Ljubica Oparnica ${ }^{\dagger}$ Stevan Pilipović ${ }^{\ddagger}$
}

\begin{abstract}
We analyze solvability of a special form of distributed order fractional differential equations

$$
\int_{0}^{2} \phi_{1}(\gamma) D^{\gamma} y(t) d \gamma=\int_{0}^{2} \phi_{2}(\gamma) D^{\gamma} z(t) d \gamma, \quad t>0
$$

within $\mathcal{S}_{+}^{\prime}$, the space of tempered distributions supported by $[0, \infty)$.

Keywords: Distributed order fractional differential equations; Tempered distribution; Laplace transform

AMS Subject 26A33; 46F12
\end{abstract}

\section{Motivation and Introduction}

We consider a distributed order fractional differential equation (1) which arises in the theory of constitutive equations for viscoelastic bodies. $\phi_{1}, \phi_{2}$ are certain functions or distributions which characterize a material under consideration and, in general, are determined from experiments. $D^{\gamma}, \gamma \in \mathbb{R}$, is the left RiemannLiouville operator of fractional differentiation or integration defined as follows.

Denote by $L_{l o c+}^{1}(\mathbb{R})$ the space of locally integrable functions $y$ on $\mathbb{R}$ such that $y(t)=0, t<0$. Then for $y \in L_{l o c+}^{1}(\mathbb{R})$ the left fractional integral of order $\gamma>0$ is defined by

$$
I^{\gamma} y(t):=\frac{1}{\Gamma(\gamma)} \int_{0}^{t}(t-\tau)^{\gamma-1} y(\tau) d \tau, \quad t>0
$$

Here $\Gamma$ is the Euler gamma function. If $\gamma=0$ then $I^{0} y:=y$. It can be shown (cf. [12]) that for $y \in L_{\text {loc }}^{1}(\mathbb{R}) \lim _{\gamma \rightarrow 0} I^{\gamma} y(t)=y(t), t \in \mathbb{R}$ almost everywhere.

Denote by $A C^{k}\left(\overline{\mathbb{R}}_{+}\right)$the space of functions $y$ such that $y$ has continuous derivatives on $\overline{\mathbb{R}}_{+}=\{x \in \mathbb{R} ; x \geq 0\}$ up to the order $k-1$ and $k$-th derivative is locally integrable function. We extend such functions to $\mathbb{R}$ so that $y(t)=0$, $t<0$.

\footnotetext{
*Faculty of Technical Sciences, University of Novi Sad, Trg D. Obradovića 5, 21000 Novi Sad, Serbia, (atanackovic@uns.ns.ac.yu)

$\dagger$ Institute of Mathematics, Serbian Academy of Science, Kneza Mihaila 35, 11000 Belgrade, Serbia, (1jubicans@sbb.co.yu)

${ }^{\ddagger}$ Department of Mathematics and Informatics, University of Novi Sad, Trg Dositeja Obradovića 4, 21000 Novi Sad, Serbia, (pilipovic@im.ns.ac.yu)
} 
Let $y \in A C^{k}\left(\overline{\mathbb{R}}_{+}\right)$. Riemann-Liouville's fractional derivative of order $\gamma \geq 0$, $\gamma \leq k$ for some $k \in \mathbb{N}$, is defined by

$$
D^{\gamma} y(t):=\frac{d^{k}}{d t^{k}} I^{k-\gamma} y(t), \quad t>0
$$

It follows that $D^{\gamma} y \in L_{l o c+}^{1}(\mathbb{R})$. We refer to Section 2 for the definition of $D^{\gamma} y$, $y \in \mathcal{S}_{+}^{\prime}$ and $\gamma \in \mathbb{R}$.

Note that $D^{\gamma} I^{\gamma} y=y$ for $y \in L_{\text {loc+ }}^{1}(\mathbb{R})$ and $I^{\gamma} D^{\gamma} y=y, \gamma>0$ in the sense of tempered distribution. We sometimes denote $D^{-\gamma} y=I^{\gamma} y, \gamma>0$.

Let $\phi$ be continuous function in $[c, d] \subset[0, k], k \in \mathbb{N}$. Distributed order fractional derivative of $y \in A C^{k}\left(\overline{\mathbb{R}}_{+}\right)$is given by

$$
\int_{c}^{d} \phi(\gamma) D^{\gamma} y(t) d \gamma
$$

Equation (1) models various physical processes. For example, if it models a viscoelastic body then (11) represents a constitutive equation of a material and connects strain $y(t)$ with corresponding stress $z(t)$ at time instant $t \geq 0$. For a standard linear viscoelastic body the constitutive equation is given as

$$
y(t)+b y^{(1)}(t)=z(t)+a z^{(1)}(t)
$$

where $(\cdot)^{(1)}=\frac{d}{d t}(\cdot)$ and $a, b$ are experimentally determined constants with the restriction $0<a<b$ following from the second law of thermodynamics. A slight generalization (see [2] and references there) of this equation is achieved by replacing the first derivative by a derivative of real order $\alpha>0$

$$
y(t)+b D^{\alpha} y(t)=z(t)+a D^{\alpha} z(t),
$$

where, again $0<a<b$. If $0<\alpha<1$ then (2) represents visco-elastic effects while for $1<\alpha<2$, (2) describes visco-inertial effects of a material. Standard procedure in building rheological models is to use more then one derivative on each side of constitutive equation. When this is done (2) becomes

$$
\sum_{n=0}^{N} b_{n} D^{\beta_{n}} y(t)=\sum_{m=0}^{M} a_{m} D^{\alpha_{m}} z(t),
$$

where $M, N \in \mathbb{N}, a_{m}, b_{n} \in \mathbb{R}$ and $\alpha_{m}, \beta_{n} \in \mathbb{R}, 0 \leq \alpha_{m}, \beta_{n} \leq 2$.

Equation (3) is interpreted in [1] as a Riemann sum. Moreover, in [1] is proposed the constitutive equation of a linear visco-elastic body in a "distributed" order form as

$$
\int_{0}^{2} \phi_{1}(\gamma) D^{\gamma} y(t) d \gamma=\int_{0}^{2} \phi_{2}(\gamma) D^{\gamma} z(t) d \gamma, \quad t>0 .
$$

In model (4) all derivatives of the stress $D^{\gamma} z$ depend on all derivatives of the strain $D^{\gamma} y$ for $\gamma \in[c, d]$. Since the upper bound in integrals in (4) is two, both, visco-elastic and visco-inertial effects are included. The presence of integral on the left hand side indicates, as experiments show, that dissipation properties depend on the order of the derivative. The integral on the right hand side is a consequence of the known principle of equipresence. 
In this paper we are looking for an $\mathcal{S}_{+}^{\prime}$ solution $z$ to (44) for a given but arbitrary $y \in \mathcal{S}_{+}^{\prime}$. Such solution will be used in [6] for solving a differential equation of motion coupled with constitutive equation (4).

In Section 2 we extend results obtained in [5] concerning integral in (4). Afterwards we define distributed order fractional derivative in $\mathcal{S}_{+}^{\prime}$ and derive it's main properties. In Section 3 we state without proof (which is given in [6]) a theorem on the existence and uniqueness of a solution to a linear fractional differential equation in the frame of $\mathcal{S}_{+}^{\prime}$. Also we derive properties of such solution. In Section 4 we connect condition for the uniqueness with a dissipation inequality that guarantees physical admissability of a equation in (4).

We note that the models with distributed order derivatives were analyzed for example in [3, 8, 7, 10, 11.

\section{Distributed order fractional derivative}

We denote by $\mathcal{S}(\mathbb{R})$ the space of rapidly decreasing functions in $\mathbb{R}$ and by $\mathcal{S}^{\prime}(\mathbb{R})$ its dual, the space of tempered distributions; $\mathcal{S}_{+}^{\prime}(\mathbb{R})$ denotes its subspace consisting of distributions supported by $[0, \infty)$. In the sequel we drop $\mathbb{R}$ in the notation. We consider in $\mathcal{S}_{+}^{\prime}$ the family

$$
f_{\alpha}(x)=\left\{\begin{array}{cc}
H(x) \frac{x^{\alpha-1}}{\Gamma(\alpha)}, & x \in \mathbb{R}, \alpha>0, \\
\frac{d^{N}}{d x^{N}} f_{\alpha+N}(x), & \alpha \leq 0, \alpha+N>0, N \in \mathbb{N},
\end{array}\right.
$$

where $H$ is Heaviside's function. It is known that $f_{\alpha} * f_{\beta}=f_{\alpha+\beta}, \alpha, \beta \in \mathbb{R}$. The convolution operator $f_{\alpha} *$ in $\mathcal{S}_{+}^{\prime}$ is the operator of fractional differentiation for $\alpha<0$ and of fractional integration for $\alpha>0$. It coincides with the operator of derivation for $-\alpha \in \mathbb{N}$ and integration for $\alpha \in \mathbb{N}$. Let $\alpha>0$ and $y \in L_{\text {loc }+}^{1}(\mathbb{R})$. Then $I^{\alpha} y=f_{\alpha} * y$. Let $y \in A C^{k}\left(\overline{\mathbb{R}}_{+}\right)$and $0<\alpha \leq k$. Then $D^{\alpha} y=f_{-\alpha} * y$.

Recall, if $y \in \mathcal{S}_{+}^{\prime}$ then its Laplace transform is defined by

$$
\widehat{y}(s)=\mathcal{L} y(s)=\left\langle y(t), \varphi(t) e^{-s t}\right\rangle, \quad \operatorname{Re} s>0, \quad \alpha \in \mathbb{R}
$$

where $\varphi \in C^{\infty}, \varphi=1$ on $(-a, \infty)$ and $\varphi=0$ in $(-\infty,-2 a), a>0$. Note that $\mathcal{L} y$ is an analytic function for $\operatorname{Re} s>0$ and that the definition of $\mathcal{L} y$ does not depend on a chosen function $\varphi$ with given proprieties. We will often use the identity

$$
\mathcal{L}\left(f_{\alpha} * y\right)(s)=\frac{1}{s^{\alpha}} \hat{y}(s), \quad \operatorname{Re} s>0 .
$$

First we analyze integral $\int_{\operatorname{supp} \phi} \phi(\gamma) D^{\gamma} y(\cdot) d \gamma$. To do this we examine the mapping $\alpha \mapsto D^{\alpha} y: \mathbb{R} \rightarrow \mathcal{S}_{+}^{\prime}$, for given $y \in \mathcal{S}_{+}^{\prime}$ (in [5] we have considered $\left.y \in L_{l o c}^{1}(\mathbb{R}) \cap \mathcal{S}_{+}^{\prime}\right)$.

Proposition 2.1. (a) Let $\alpha \in \mathbb{R}$ be fixed. Then the mapping $y \mapsto D^{\alpha} y$ is linear and continuous from $\mathcal{S}_{+}^{\prime}$ to $\mathcal{S}_{+}^{\prime}$.

(b) Let $y \in \mathcal{S}_{+}^{\prime}$ be fixed. Then $\alpha \mapsto D^{\alpha} y$ is a smooth mapping from $\mathbb{R}$ to $\mathcal{S}_{+}^{\prime}$.

(c) The mapping $(\alpha, y) \mapsto D^{\alpha} y$ is continuous from $\mathbb{R} \times \mathcal{S}_{+}^{\prime}$ to $\mathcal{S}_{+}^{\prime}$.

Proof. (a) The continuity of $y \mapsto D^{\alpha} y=f_{-\alpha} * y$ is clear since for $g \in \mathcal{S}_{+}^{\prime}$, $f \mapsto f * g$ is a continuous mapping of $\mathcal{S}_{+}^{\prime}$ into $\mathcal{S}_{+}^{\prime}$. 
(b) It is known that there exists a continuous function $F$, supp $F \subset[0, \infty)$ and $k \in \mathbb{N}$ such that $|F(x)|<C(1+|x|)^{k}, x \in \mathbb{R}$ and $y=D^{k} F$. So the mapping $\alpha \mapsto D^{\alpha} y$ equals $\alpha \mapsto D^{\alpha+k} F$. By [5, Proposition 1] we know that for fixed $k$ and $\alpha \in \mathbb{R}, \alpha+k \mapsto D^{\alpha+k} F$ is smooth so the same hold for $\alpha \mapsto D^{\alpha+k} F$.

(c) Since $\mathcal{S}$ is Fréchet space as well as locally convex, the separate continuity proved in (a) and (b) imply joint continuity (c.f. [13, Corollary to Theorem 34.1]).

By $\mathcal{E}^{\prime}(\mathbb{R})$ is denoted the space of compactly supported distributions i.e. the dual space of $\mathcal{E}(\mathbb{R})=C_{0}^{\infty}(\mathbb{R})$.

Definition 2.2. Let $\phi \in \mathcal{E}^{\prime}(\mathbb{R})$ and $y \in \mathcal{S}_{+}^{\prime}$. Then $\int_{\operatorname{supp} \phi} \phi(\gamma) D^{\gamma} y d \gamma$ is defined as an element of $\mathcal{S}_{+}^{\prime}$ by

$$
\left\langle\int_{\operatorname{supp} \phi} \phi(\gamma) D^{\gamma} y(t) d \gamma, \varphi(t)\right\rangle=\left\langle\phi(\gamma),\left\langle D^{\gamma} y(t), \varphi(t)\right\rangle\right\rangle, \quad \varphi \in \mathcal{S}(\mathbb{R})
$$

Such defined distribution is called distributed order fractional derivative.

By Proposition 2.1, part (b), $\gamma \mapsto D^{\gamma} y: \mathbb{R} \rightarrow \mathcal{S}_{+}^{\prime}$ is smooth as well as $\gamma \mapsto\left\langle D^{\gamma} y(t), \varphi(t)\right\rangle: \mathbb{R} \rightarrow \mathbb{R}$. Since $\mathcal{S}$ is a Frećhet space it follows that in it's dual space the strong and weak boundedness are the same, thus a linear functional defined by (6) is continuous from $\mathcal{S}$ to $\mathbb{C}$ and therefore is a tempered distribution supported by $[0, \infty)$.

The following two examples are often used in applications.

Example 2.3. a) Let $\gamma_{i} \in \mathbb{R}, i \in\{0,1, . ., k\}$ and $\phi(\cdot)=\sum_{i=0}^{k} a_{i} \delta^{(i)}\left(\cdot-\gamma_{i}\right)$. Then (6) gives

$$
\int_{\operatorname{supp} \phi} \phi(\gamma) D^{\gamma} y(\cdot) d \gamma=\sum_{i=0}^{k} a_{i} D^{\gamma_{i}} y(\cdot), \quad \text { in } \mathcal{S}_{+}^{\prime}
$$

b) Let $\phi$ be a continuous function in $[c, d] \subset \mathbb{R}$ for some $c<d$, then

$$
\int_{c}^{d} \phi(\gamma) D^{\gamma} y(\cdot) d \gamma=\lim _{N \rightarrow \infty} \sum_{i=1}^{N} \phi\left(\gamma_{i}\right) D^{\gamma_{i}} y(\cdot) \Delta \gamma_{i}, \quad \text { in } \mathcal{S}_{+}^{\prime},
$$

where $\gamma_{i}$ are points of interval $[c, d]$ in usual definition of the Riemann sum for the integral.

Proposition 2.4. Let $\phi \in \mathcal{E}^{\prime}(\mathbb{R})$ and $y \in \mathcal{S}_{+}^{\prime}$. Then:

a)

$$
y \mapsto \int_{\operatorname{supp} \phi} \phi(\gamma) D^{\gamma} y d \gamma
$$

is a linear and continuous mapping from $\mathcal{S}_{+}^{\prime}$ to $\mathcal{S}_{+}^{\prime}$.

b)

$$
\mathcal{L}\left(\int_{\operatorname{supp} \phi} \phi(\gamma) D^{\gamma} y d \gamma\right)(s)=\hat{y}(s)\left\langle\phi(\gamma), s^{\gamma}\right\rangle, \quad \text { Res }>0
$$


c) If $\phi$ is continuous function on $[c, d]$ and $\phi(\gamma)=0$ for $\gamma \notin[c, d]$ then

$$
\mathcal{L}\left(\int_{c}^{d} \phi(\gamma) D^{\gamma} y d \gamma\right)(s)=\hat{y}(s) \int_{c}^{d} \phi(\gamma) s^{\gamma} d \gamma, \quad \text { Res }>0 .
$$

Proof. a) Clearly, this mapping is linear. Let $y_{n} \rightarrow 0$ in $\mathcal{S}_{+}^{\prime}$. Then $\left\langle\phi(\gamma),\left\langle D^{\gamma} y_{n}, \varphi\right\rangle\right\rangle \rightarrow$ 0 , as $n \rightarrow \infty$, since by Proposition 2.1 part (a), $\left\langle D^{\gamma} y_{n}, \varphi\right\rangle \rightarrow 0$, as $n \rightarrow \infty$.

b) By the definition,

$$
\begin{aligned}
& \mathcal{L}\left(\int_{\operatorname{supp} \phi} \phi(\gamma) D^{\gamma} y d \gamma\right)(s)=\left\langle\int_{\operatorname{supp} \phi} \phi(\gamma) D^{\gamma} y(t) d \gamma, \varphi(t) e^{-s t}\right\rangle \\
& \quad=\left\langle\phi(\gamma),\left\langle D^{\gamma} y(t), \varphi(t) e^{-s t}\right\rangle\right\rangle=\left\langle\phi(\gamma), s^{\gamma} \hat{y}(s)\right\rangle, \quad \operatorname{Re} s>0 .
\end{aligned}
$$

c) In the case that $\phi$ is continuous we have

$$
\left\langle\phi(\gamma), s^{\gamma} \hat{y}(s)\right\rangle=\int_{\operatorname{supp} \phi} \phi(\gamma) s^{\gamma} \hat{y}(s) d \gamma
$$

and therefore the assertion follows.

If we assume that $y, z \in \mathcal{S}_{+}^{\prime}$ in (4), put $\phi=\phi_{2}$ and $g=\int_{\operatorname{supp} \phi_{1}} \phi_{1}(\gamma) D^{\gamma} z$ then the solvability of (4) with respect to $z$ reduces to the solvability of

$$
\int_{\operatorname{supp} \phi} \phi(\gamma) D^{\gamma} z=g, \quad g \in \mathcal{S}_{+}^{\prime}
$$

\section{Linear fractional differential equation in $\mathcal{S}_{+}^{\prime}$}

Assuming that $g \in \mathcal{S}_{+}^{\prime}$ and that $\phi$ is of the form as in Example 2.3. equation (7) becomes

$$
\sum_{i=0}^{k} a_{i} D^{\gamma_{i}} z=g, \quad \text { in } \mathcal{S}_{+}^{\prime}
$$

We suppose that $\gamma_{i} \in[0,2)$ such that $\gamma_{0}>\gamma_{i}>\gamma_{i+1}>\gamma_{k}, i \in\{1, \ldots k-1\}$.

Theorem 3.1. Equation (8) has a unique solution $z \in \mathcal{S}_{+}^{\prime}$ if and only if

$$
\left(A_{0}\right) \quad \sum_{i=0}^{k} a_{i} s^{\gamma_{i}} \neq 0, \quad s \in \mathbb{C}_{+}=\{s \in \mathbb{C} ; \text { Re } s>0\} .
$$

The proof is given in [6. The solution to (8) that is obtain in Theorem 3.1 is given by $z=l * g$, where

$$
l(t)=\mathcal{L}^{-1}\left(\frac{1}{\sum_{i=0}^{k} a_{i} s^{\gamma_{i}}}\right)(t), \quad t>0
$$

is a fundamental solution to (8) i.e. solution to $\sum_{i=0}^{k} a_{i} D^{\gamma_{i}} y=\delta$.

The following lemma gives main properties of $l$ defined by (9). 
Lemma 3.2. Assume $\left(A_{0}\right)$. Let $\gamma_{i} \in[0,2)$ and $\gamma_{0}>\gamma_{i}>\gamma_{i+1}>\gamma_{k}$, for all $i \in\{1, . ., k-1\}$. Let $l$ be defined by (9) and $l(t)=0, t<0$. Then:

(i) $l$ is a locally integrable function in $\mathbb{R}$.

(ii) Moreover, $l$ is absolutely continuous in $\mathbb{R}$, if $\gamma_{0}-\gamma_{k}>1$.

Proof. (i) Let $\gamma_{k}=0$ and $a_{k} \neq 0$. Consider the integral

$$
\int_{\Gamma} \frac{e^{s t} d s}{\sum_{i=0}^{k} a_{i} s^{\gamma_{i}}}, \quad t>0
$$

where $\Gamma=\bigcup_{i=1}^{5} \Gamma_{i}$ and for arbitrarily chosen $R>0,0<\varepsilon<R$ and $x_{0}>0, \Gamma_{i}$ are given by

$$
\begin{gathered}
\Gamma_{0}:\left\{z ; \operatorname{Re} z=x_{0} ; 0<\arg z<\phi_{0}=\arcsin \frac{x_{0}}{R}\right\} ; \\
\Gamma_{1}: z=R e^{i \varphi},-\phi_{0}<\phi_{0} \leq \varphi<\pi ; \quad \Gamma_{2}: z=R e^{i \varphi},-\pi<\varphi \leq-\phi_{0}<0 ; \\
\Gamma_{3}: z=\varepsilon e^{i \varphi},-\pi<\varphi<\pi ; \quad \Gamma_{4}: z=x e^{i \pi} ; \quad \Gamma_{5}: z=x e^{-i \pi}, \quad x \in(\varepsilon, R) .
\end{gathered}
$$

By the Cauchy residue theorem, letting $\varepsilon \rightarrow 0$ and $R \rightarrow \infty$, one obtains

$$
l(t)=\sum_{s=s_{m}, m=1}^{n} \operatorname{Res}\left\{\frac{e^{s t}}{\sum_{i=0}^{k} a_{i} s^{\gamma_{i}}}\right\}+l_{0}(t), \quad t>0
$$

where $s_{m}$ are poles of the function $s \mapsto \frac{e^{s t}}{\sum_{i=0}^{k} a_{i} s^{\gamma_{i}}}$ and

$$
l_{0}(t)=\frac{1}{\pi} \int_{0}^{\infty} e^{-s t} r(s) d s, \quad t>0,
$$

with

$$
r(s)=\frac{2 \sum_{i=0}^{k} a_{i} s^{\gamma_{i}} \sin \left(\gamma_{i} \pi\right)}{\sum_{i=0}^{k} a_{i}^{2} s^{2 \gamma_{i}}+2 \sum_{i, j=0, i \neq j}^{k} a_{i} a_{j} s^{\gamma_{i}+\gamma_{j}} \cos \left(\gamma_{i}-\gamma_{j}\right) \pi}, s>0 .
$$

We refer to 4 for similar calculations.

Let $0 \leq a \leq b$. Then

$$
\int_{a}^{b} l_{0}(t) d t=\int_{0}^{\infty}\left(e^{-s a}-e^{-s b}\right) \frac{1}{s} r(s) d s .
$$

Since $\gamma_{k}=0$, this integral is finite in a neighborhood of $s=0$. In a neighborhood of $s=\infty$ we have $\frac{r(s)}{s} \sim \frac{1}{s^{\gamma}+\mathrm{r}}$. Thus $\gamma_{0}+1>1$ implies that the integral in (12) is finite. Therefore, $l_{0}$ is locally integrable. By (10) and the fact that Re $s_{m}<0$ (by $\left(A_{0}\right)$ ), we obtain that $l$ is locally integrable.

Let $\gamma_{k}>0$. Then

$$
l=\mathcal{L}^{-1}\left(\frac{1}{s^{\gamma_{k}}}\right) * \mathcal{L}^{-1}\left(\frac{1}{\sum_{i=0}^{k} a_{i} s^{\gamma_{i}-\gamma_{k}}}\right)=f_{\gamma_{k}} * l_{1},
$$

where

$$
l_{1}=\mathcal{L}^{-1}\left(\frac{1}{\sum_{i=0}^{k} a_{i} s^{\beta_{i}}}\right), \quad \beta_{i}=\gamma_{i}-\gamma_{k}, \quad i \in\{0,1, . ., k\}, \quad \text { Re } s>0,
$$


and $f_{\gamma_{k}}$ is defined by (5). Note that $\beta_{k}=0$. By the first part of the proof, $l_{1}$ is a locally integrable function. Since $f_{\gamma_{k}}$ is locally integrable, $l$ is locally integrable as the convolution of two locally integrable functions.

(ii) Let $\gamma_{k}=0$. Then (11) is finite in a neighborhood of $s=0$. In a neighborhood of $s=\infty$ we have that $r(s) \sim \frac{1}{s \gamma_{0}}$. Since $\gamma_{0}>1$, the integrand in (11) is integrable for all $t>0$ and (11) is finite. Let $t_{0}>0$. Since $\left|e^{-s t} r(s)\right| \leq$ $e^{-s t_{0}}|r(s)|:=g(s)$ for all $t>t_{0}$ and $g \in L^{1}((0, \infty))$, by the classical theory we obtain that (11) defines a continuous function for $t>t_{0}$. It follows that $l_{0}$ and $l$ (by (10)) are continuous for $t>0$. Further on, since $\left|\partial_{t}\left(e^{-s t} r(s)\right)\right| \leq$ $e^{-s t_{0}}|\operatorname{sr}(s)|:=g_{1}(s)$, for all $t>t_{0}$ and $g_{1} \in L^{1}(0, \infty)$, we obtain that $l_{0}$ is differentiable and

$$
l_{0}^{\prime}(t)=\int_{0}^{\infty}(-s) e^{-s t} r(s), t>0
$$

Since $-s r(s) \sim \frac{1}{s \gamma_{0}-1}$ in a neighborhood of $s=\infty$, as in (i) we show that $l_{0}^{\prime}$ is a locally integrable function. Therefore, the derivative $l_{0}^{\prime}$ exists and it is a locally integrable function. It means that $l$ is absolutely continuous.

For $\gamma_{k}>0$ we proceed as in (i) and obtain $l=f_{\gamma_{k}} * l_{1}$ with $l_{1}$ absolutely continuous. Therefore, $l$ is also absolutely continuous.

Remark 3.3. If $\gamma_{i} \in[0, \infty)$ and if $\gamma_{0}>p, p \in \mathbb{N}$, then $l$ is continuous in $\mathbb{R}$ as well as its derivatives up to order $p-1$ while the $p$-th derivative is a locally integrable function, i.e. $l \in A C^{p}$.

\section{Comments from mechanics and further appli- cations}

Equation (8) represents a constitutive equation of a visco-elastic body. We will show that in the case when there exist $s_{0} \in \mathbb{C}_{+}$such that $\sum_{i=0}^{k} a_{i} s_{0}^{\gamma_{i}}=0$ it follows that the dissipation inequality (14), (see [9]) is violated. The dissipation inequality requires that for any $T>0$, any $y$ and $z$ the solution to $\sum_{i=0}^{k} a_{i} D^{\gamma_{i}} z(t)=y(t), t>0$, the dissipation work, $A_{d}$ is nonnegative, i.e.

$$
A_{d}=\int_{0}^{T} z(t) y^{(1)}(t) d t \geq 0 .
$$

Let $T>0$ and $y(t)=H(t)-H(t-\tau), 0<\tau<T, t>0$. Then

$$
z(t)=\mathcal{L}^{-1}\left(\frac{1}{s} \frac{1}{\sum_{i=0}^{k} a_{i} s^{\gamma_{i}}}-\frac{1}{s} \frac{e^{-\tau s}}{\sum_{i=0}^{k} a_{i} s^{\gamma_{i}}}\right)=\int_{0}^{t} g(u) d u, \quad t>0,
$$

where $g(u)=l(u)-l(u-\tau)$ and $l$ is the fundamental solution to (8) given by (9). Assume that $w\left(s_{0}\right)=\sum_{i=0}^{k} a_{i} s_{0}^{\gamma_{i}}=0$ for $s_{0}=u+i v, u>0$. Then by (10)

$$
l(t)=\left.\frac{e^{s t}}{w^{(1)}(s)}\right|_{s=s_{0}}+\left.\sum_{j=1}^{k}\left(\frac{e^{s t}}{w^{(1)}(s)}\right)\right|_{s=s_{j}}+l_{0}(t), \quad t>0 .
$$

Further, note that

$$
\left.\frac{e^{s t}}{w^{(1)}(s)}\right|_{s=s_{0}}=\frac{e^{u t}[\cos (v t)+i \sin (v t)]}{w^{(1)}\left(s_{0}\right)}, \quad t>0,
$$


represents oscillations with increasing amplitudes. Inserting (15) in (14) we obtain

$$
A_{d}=\lim _{t \rightarrow 0} \int_{0}^{t} g(u) d u-\int_{0}^{\tau} g(u) d u \geq 0, \quad \tau, t>0 .
$$

It is obvious that due to the presence of the term (16) in $g(t)$ the inequality (17) could be violated by a suitable choice of $\tau$.

\section{References}

[1] T. M. Atanackovic, A generalized model for the uniaxial isothermal deformation of a viscoelastic body, Acta Mech. 159 (2002), pp. 77-86.

[2] T. M. Atanackovic, A modified Zener model of a viscoelastic body, Continuum Mech. Thermodyn. 14 (2002), pp. 137-148.

[3] T. M. Atanackovic, On a distributed derivative model of viscoelastic body, CRAS Mechanics. 331 (2003), pp. 687-692.

[4] T. M. Atanackovic, LJ. Oparnica, and S. Pilipović, On a model of viscoelastic rod in unilateral contact with a rigid wall, IMA J. Appl. Math. 1 (2005), $1-13$.

[5] T. M. Atanackovic, LJ. Oparnica, and S. Pilipović, On a nonlinear distributed order fractional differential equation J. Math. Anal. Appl. 328 (2007), pp. 590-608.

[6] T. M. Atanackovic, LJ. Oparnica, and S. Pilipović, Semilinear ordinary differential equation coupled with distributed order fractional differential equation, preprint (2008), arXiv:0811.2871v1

[7] T. M. Atanackovic and S. Pilipović, On a class of equations arising in linear viscoelasticity theory, ZAMM, 85 (2005), pp. 748-754.

[8] M. Caputo, Distributed order differential equation modeling dialectric induction and diffusion, Fract. Calc. Appl. Anal. 4 (2001), pp. 421-442.

[9] R. Christensen, Theory of Viscoelasticity, Academic Press, New York, 1982.

[10] F. Mainardi, G. Pagnini, and R. Gorenflo, Some aspects of fractional diffusion equation of single and distributed order, Appl. Math. Comput. 187 (2006), pp. 295-305.

[11] A. Nakhushev, Fractional Calculus and its Applications, Fizmatlit, Moscow, 2003.

[12] S. G. Samko, A. Kilbas, and O.I. Marichev, Fractional Integrals and Derivatives, Theory and Applications, Gordon and Breach Science Publishers, Amsterdam, 1993.

[13] F. Treves, Topological Vector Spaces, Distibutions and Kernels, Academic Press, New York, 1967. 